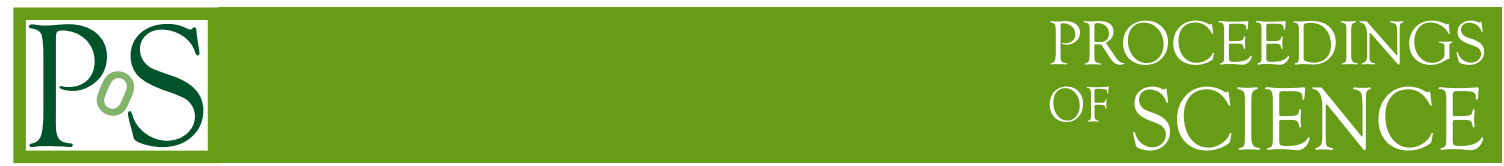

\title{
Directed, elliptic and triangular flow of D mesons in ALICE
}

\author{
Stefano Trogolo* (on behalf of the ALICE Collaboration) \\ Università degli Studi di Padova, Dip. di Fisica e Astronomia "Galileo Galilei" \\ INFN - Sez. Padova, \\ Via Marzolo 8, 35131 Padova, Italy \\ E-mail: stefano.trogolo@cern.ch
}

Heavy flavour quarks, like charm and beauty, are sensitive probes to investigate the colourdeconfined medium created in high-energy heavy-ion collisions, the quark-gluon plasma (QGP). The ALICE Collaboration measured the production of strange and non-strange $\mathrm{D}$ mesons in $\mathrm{Pb}-\mathrm{Pb}$ collisions at $\sqrt{s_{\mathrm{NN}}}=5.02 \mathrm{TeV}$. The measurement of elliptic $\left(v_{2}\right)$ and triangular $\left(v_{3}\right)$ flow of D mesons gives insight into the participation of the charm quark in the collective motion of the medium at low transverse momentum $\left(p_{\mathrm{T}}\right)$, while it constrains the path-length dependence of in-medium energy loss. Furthermore, the coupling of the charm quark to the light quarks in the underlying medium is investigated with the event-shape engineering (ESE) technique to the non-strange D-meson elliptic flow. Finally, the effect of the magnetic field created in the early stage of the collisions is studied via the first measurement at LHC energies of the charge-dependent directed flow of $\mathrm{D}^{0}$ as a function of pseudorapidity.

HardProbes 2020

1-6 June 2020

Austin, Texas

${ }^{*}$ Speaker 


\section{Introduction}

In ultra-relativistic heavy-ion collisions, heavy quarks are largely produced via hard-scattering processes on a timescale shorter than the quark-gluon plasma (QGP) formation time. Therefore they experience the full system evolution and interact with the medium constituents via elastic and inelastic processes. In particular, the measurement of the azimuthal anisotropies can provide insights into the interaction of heavy quarks with the QGP and the initial condition of the system. They are characterised by the magnitude of the Fourier coefficiencts $v_{n}=\left\langle\cos \left[\mathrm{n}\left(\varphi-\Psi_{\mathrm{n}}\right)\right]\right\rangle$, where $\varphi$ is the azimuthal angle of the particle and $\Psi_{\mathrm{n}}$ is the symmetry plane angle relative to the $\mathrm{n}^{\text {th }}$ harmonic.

The first harmonic coefficient $v_{1}$, known as directed flow, is suggested to be a useful probe of the strong initial magnetic field created in the collision that induces electromagnetic currents in the QGP. Indeed, the early production of charm quarks and their relaxation time are similar to the QGP lifetime and therefore charmed hadrons experience the evolution of the magnetic field. The expected consequence of these currents is a charge-dependent contribution to the pseudorapidityodd component of $v_{1}$ for $\mathrm{c}$ and $\overline{\mathrm{c}}$ quarks. The second order flow coefficient $v_{2}$, called elliptic flow, is the dominant component in non-central collisions and, at low $p_{\mathrm{T}}$ is sensitive to the coupling of heavy quarks with the underlying medium, while at high $p_{\mathrm{T}}$ can constrain the path-length dependence of the in-medium energy loss. The third coefficient $v_{3}$, called triangular flow, originates from event-by-event fluctuations in the initial distribution of gluons and nucleons in the overlap region of the collision and therefore it is sensitive to the initial conditions of the system. Further insights into the dynamics of heavy quarks in the medium is reached with the application of the event-shape engineering (ESE) technique, which allows to investigate the coupling between charm quarks and light flowing quarks of the bulk.

Charmed mesons were reconstructed in ALICE at midrapidity $(|y|<0.8)$ via the decay channels $\mathrm{D}^{0} \rightarrow \mathrm{K}^{-} \pi^{+}, \mathrm{D}^{+} \rightarrow \mathrm{K}^{-} \pi^{+} \pi^{+}, \mathrm{D}^{*+} \rightarrow \mathrm{D}^{0} \pi^{+}$, and $\mathrm{D}_{\mathrm{s}}^{+} \rightarrow \phi \pi^{+} \rightarrow \mathrm{K}^{-} \mathrm{K}^{+} \pi^{+}$and their charge conjugates. The combinatorial background was reduced by applying geometrical selections on the decay-vertex topology and particle identification on the daughters tracks. A simultaneous fit to the
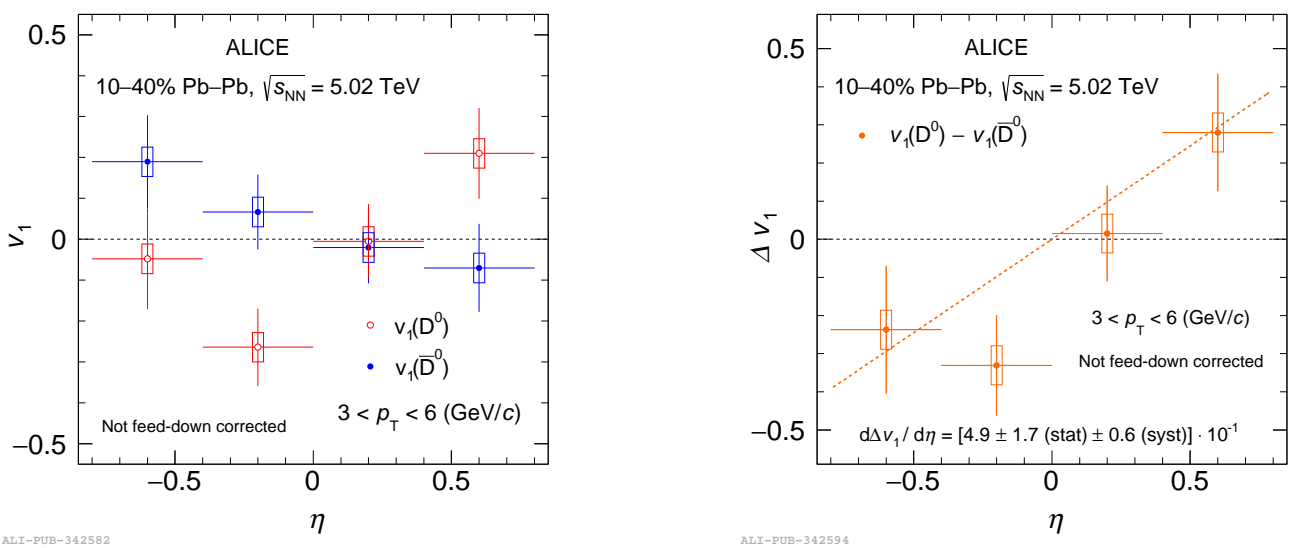

Figure 1: $\mathrm{D}^{0}$ and $\overline{\mathrm{D}}^{0} v_{1}^{\text {odd }}$ (left) and their difference $\Delta v_{1}^{\text {odd }}$ (right) as a function of pseudorapidity in the $10-40 \%$ centrality class. 


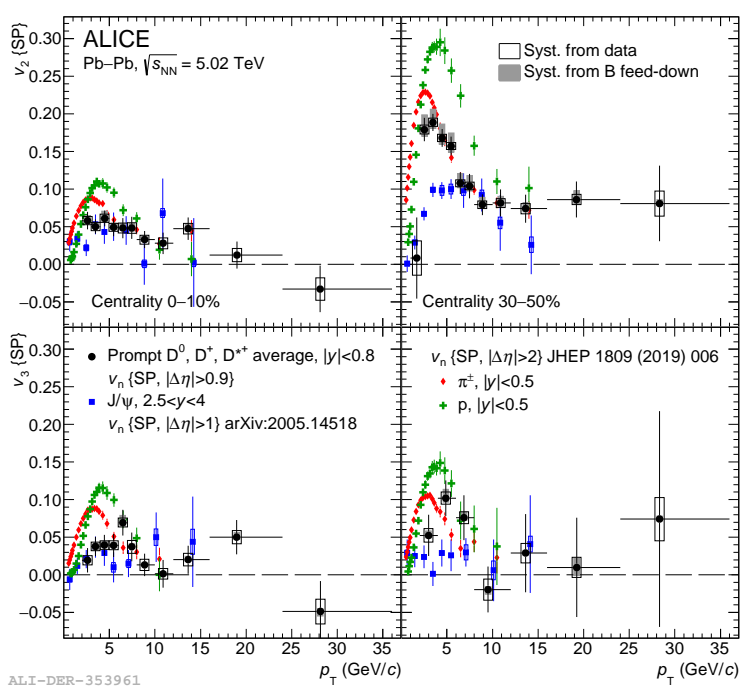

Figure 2: Average $v_{2}$ (top) and $v_{3}$ (bottom) of prompt $\mathrm{D}^{0}, \mathrm{D}^{+}$, and $\mathrm{D}^{*+}$ in central (left) and mid-central (right) $\mathrm{Pb}-\mathrm{Pb}$ collisions at $\sqrt{s_{\mathrm{NN}}}=5.02 \mathrm{TeV}$ as a function of $p_{\mathrm{T}}$. The results are compared with $v_{2}$ and $v_{3}$ of $\pi^{ \pm}, \mathrm{p}+\overline{\mathrm{p}}[7]$ and inclusive $\mathrm{J} / \psi$ mesons [8] measured at the same centre-of-mass energy

invariant mass distribution and the $v_{\mathrm{n}}$ as a function of the invariant mass is performed in order to obtain the flow coefficient of the signal $[2,3]$. The fraction of promptly produced D mesons was estimated with a theory-driven approach based on FONLL calculation [4, 5].

In this proceeding, the most recent results on the azimuthal anisotropy of strange and non strange $\mathrm{D}$ mesons, measured in $\mathrm{Pb}-\mathrm{Pb}$ collisions at $\sqrt{s_{\mathrm{NN}}}=5.02 \mathrm{TeV}$, are presented. In particular the $v_{1}$ measurement [6] was performed with the data sample collected in 2015 , which consisted of $10^{8}$ minimum bias collisions, corresponding to $\simeq 13 \mu \mathrm{b}^{-1}$. The dataset collected in 2018 was used for the $v_{2}$ and $v_{3}$ measurements [3] and consisted of $100 \times 10^{6}$ and $85 \times 10^{6}$ events in the $0-10 \%$ and $30-50 \%$ centrality class, corresponding to an integrated luminosity of $\simeq 130 \mu \mathrm{b}^{-1}$ and $\simeq 56 \mu \mathrm{b}^{-1}$ respectively.

\section{Directed flow}

The directed flow was measured separately for $\mathrm{D}^{0}$ and $\overline{\mathrm{D}}^{0}$ with the scalar-product method in the $10-40 \%$ centrality class. The spectator plane was reconstructed via the transverse asymmetry in the energy deposited in two neutron Zero-Degree Calorimeters (ZDCs) located at $\eta>8.8$ (ZDC-A) and $\eta<-8.8$ (ZDC-C). The $v_{1}$ rapidity-odd component was computed as $v_{1}^{\text {odd }}=\frac{1}{2}\left[v_{1}(\mathrm{~A})-v_{1}(\mathrm{C})\right]$, following the convention of a positive sign of the directed flow of spectator neutrons at positive pseudorapidity. Figure 1 shows the $v_{1}^{\text {odd }}$ of $\mathrm{D}^{0}$ and $\overline{\mathrm{D}}^{0}$ (left panel) with $3<p_{\mathrm{T}}<6 \mathrm{GeV} / c$ and the related difference $\Delta v_{1}^{\text {odd }}$ (right panel), as a function of $\eta$. A possible hint of signal is observed by fitting the difference with a linear function $\Delta v_{1}^{\text {odd }}=k \times \eta$ and obtaining a positive slope with a significance of $2.7 \sigma$, which is also similar to the one measured for the charged hadrons [6]. 


\section{Elliptic and triangular flow}

Figure 2 shows the average elliptic and triangular flow of prompt $\mathrm{D}^{0}, \mathrm{D}^{+}$, and $\mathrm{D}^{*+}$ measured in central and mid-central $\mathrm{Pb}-\mathrm{Pb}$ collisions with the scalar-product method [3]. The result is compared to that of charged pions, protons [7] and inclusive $\mathrm{J} / \psi$ [8]. The non-strange D-meson $v_{2}$ is positive for $p_{\mathrm{T}}>2 \mathrm{GeV} / c$ and increases from central to mid-central collisions, reflecting the increasing eccentricity of the interaction region. Similarly the $v_{3}$ is positive in the same $p_{\mathrm{T}}$ range, but it is compatible among the two centrality classes, as observed also for light hadrons. The comparison with other particles shows a mass ordering for $p_{\mathrm{T}}<3 \mathrm{GeV} / c$ which is explained as an interplay between anisotropic and radial flow. At intermediate $p_{\mathrm{T}}, 3-8 \mathrm{GeV} / c, v_{\mathrm{n}}(\mathrm{D}) \simeq v_{\mathrm{n}}(\pi)<v_{\mathrm{n}}(\mathrm{p})$ which is consistent with the constituent-quark scaling of $v_{\mathrm{n}}$. Furthermore, $v_{\mathrm{n}}(\mathrm{D})>v_{\mathrm{n}}(\mathrm{J} / \psi)$ in the same range and this confirms the charm-quark coalescence with light quarks. Finally, all $v_{\mathrm{n}}$ coefficients are similar for $p_{\mathrm{T}}>8 \mathrm{GeV} / c$ and this is due to a similar path-length dependence of in-medium energy loss for quarks and gluons. The non-strange D-meson $v_{2}$ and $v_{3}$ was also compared with the predictions of theoretical models and the result is well described by those including both quark coalescence and fragmentation in the hadronisation phase. In addition, it was also possible to constrain the charm spatial diffusion in the range $1.5<2 \pi T_{c} D_{s}<7$, by evaluating the agreement between the $v_{\mathrm{n}}$ coefficients and the models, and this lead to charm thermalization times $\tau_{\text {charm }}=3-14 \mathrm{fm} / \mathrm{c}$ [3].

The $\mathrm{D}_{\mathrm{s}}^{+}$elliptic flow was measured in the $30-50 \%$ centrality class with the same analysis technique used for non-strange D-meson $v_{2}$ and the results are compatible as shown in Figure 3. Furthermore, the comparison with theoretical predictions shows that models based on the charmquark transport in an hydrodynamically expanding medium can fairly well describe both strange and non-strange $v_{2}$ in mid-central collisions.

\section{Event-shape engineering}

The $v_{2}$ of $\mathrm{D}^{0}, \mathrm{D}^{+}$, and $\mathrm{D}^{*+}$ mesons was further investigated by applying the event-shape engineering technique [11], which allows to classify events with fixed centrality but different

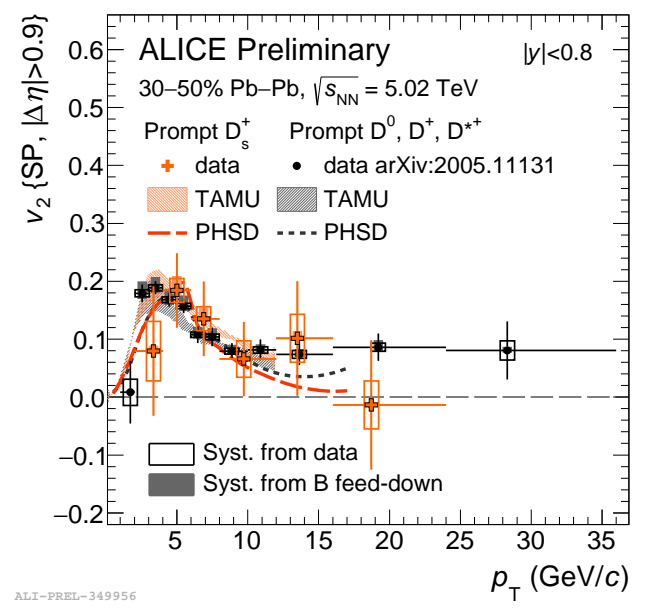

Figure 3: $\mathrm{D}_{\mathrm{s}}^{+} v_{2}$ in mid-central $\mathrm{Pb}-\mathrm{Pb}$ collisions at $\sqrt{s_{\mathrm{NN}}}=5.02 \mathrm{TeV}$ as a function of $p_{\mathrm{T}}$. The result is compared to non-strange D-meson $v_{2}$ and theoretical model predictions [9, 10]. 
average elliptic flow. The latter is quantified via the magnitude of the second-harmonic reduced flow vector $q_{2}=\left|\vec{Q}_{2}\right| / \sqrt{M}$, where $M$ is the event multiplicity and $\vec{Q}_{2}$ is the second-harmonic flow vector. The non-strange D-meson $v_{2}$ was measured in $20 \%$ of events with largest/smallest $q_{2}$ in mid-central and, for the first time, central $\mathrm{Pb}-\mathrm{Pb}$ collsions. The results, described in [3], show a decrease (increase) by about $50 \%$ in the $20 \%$ small- $q_{2}$ (large- $q_{2}$ ) class and this confirms a correlation between the D-meson azimuthal anisotropy and the collective expansion of the medium. In addition, different implementations of theoretical models give a similar description of the measured ratios, suggesting that the ESE effect is more related to the initial geometry rather than to the dynamic evolution of charm quarks in the medium.

\section{Conclusions}

The ALICE Collaboration has measured the $v_{2}$ and $v_{3}$ of prompt stange and non-strange $\mathrm{D}$ mesons and the charge-dependent $v_{1}^{\text {odd }}$ of $\mathrm{D}^{0}$ and $\overline{\mathrm{D}}^{0}$ mesons in $\mathrm{Pb}-\mathrm{Pb}$ collisions at $\sqrt{s_{\mathrm{NN}}}=5.02 \mathrm{TeV}$.

The first measurement at the LHC energies of $\mathrm{D}^{0}$ and $\overline{\mathrm{D}}^{0} v_{1}^{\text {odd }}$ as a function $\eta$ gives a hint of a charge difference and it was quantified with the slope of $\Delta v_{1}^{\text {odd }}$, which was found to be positive with a significance of $2.7 \sigma$. The D-meson $v_{2}$ and $v_{3}$ are observed to be positive for $p_{\mathrm{T}}>2 \mathrm{GeV} / c$ in central and mid-central. The ordering observed for the two harmonics as a function of $p_{\mathrm{T}}$ confirms the interplay between charm-quark transport and path-length dependence at different $p_{\mathrm{T}}$. Finally, the usage of the event-shape engineering technique in the $v_{2}$ measurement shows a positive correlation between the D-meson azimuthal anisotropy and the collective expansion of the the bulk of light quarks.

\section{References}

[1] ALICE Coll., B. B. Abelev et. al, Int. J. Mod. Phys. A 29 (2014) 1430044

[2] N. Borghini and J.-Y. Ollitrault, Phys. Rev. C 70 (2004) 064905

[3] ALICE Coll., S. Acharya et. al, arXiv:2005.11131 [nucl-ex]

[4] M. Cacciari, M. Greco and P. Nason, JHEP 9805 (1998) 007

[5] ALICE Coll., S. Acharya et. al, Phys. Rev. Lett. 120 (2018) 10, 102301

[6] ALICE Coll., S. Acharya et. al, Phys. Rev. Lett. 125 (2020) 2, 022301

[7] ALICE Coll., S. Acharya et. al, JHEP 09 (2018) 006

[8] ALICE Coll., S. Acharya et. al, arXiv:2005.14518 [nucl-ex]

[9] M. He and R. Rapp, Phys. Rev. Lett. 124 no. 4 (2020) 042301

[10] T. Song et. al, Phys. Rev.C 93 no. 3, 554 (2016) 034906

[11] J. Schukraft, A. Timmins, and S. A. Voloshin, Phys. Lett. B 719 (2013) 394 